\title{
DID THE BULGE FORM ALL AT ONCE?
}

\author{
R.M. RICH
}

Department of Astronomy, Columbia University, 538 W. 120th St. NY NY 10027

\section{Introduction}

It is reasonable to say that if Jan Oort were alive today, he would no doubt find recent developments in the study of the Galactic bulge to be fascinating. Oort considered the Galactic bulge in two contexts. First, he was interested in the use of the RR Lyrae stars as probes to determine the distance to the Galactic Center. No doubt, Oort would have been excited about the growing evidence of the bulge's triaxiality, as well as by the debate over the age of the bulge. His second interest was in the nature of activity at the center, an issue that I will not discuss in this review. The latter also remains an unsolved problem of the Milky Way, and (based on his work) one that might have been nearer to his heart than this one. Yet the question of when the bulge formed is ultimately a question about the formation history of the Galaxy. The oldest stars (those whose ages we are certain of) are found in Galactic globular clusters, the sum total of which are $\approx 5 \times 10^{7} \mathrm{M}_{\odot}$. The field population of the bulge is $\approx 2-3 \times 10^{10} \mathrm{M}_{\odot}$, an order of magnitude more massive than the field population of the metal poor spheroid. So if the bulge formed all at once, and early, then the Milky Way had a luminous, even cataclysmic youth. But if the bulge formed later in the history of our galaxy, as a starburst or dynamical instability of the central disk, then the young Milky Way may have been inconspicuous and primeval galaxies may be hard to find indeed. If our bulge formed very early, its stellar population might have much in common with the giant ellipticals, while a late bulge might teach us much about processes that affect galaxy evolution.

The question "Did the bulge form all at once?" is really the question "does the bulge have an age range?". The two extreme populations in this regard are globular clusters, which have an age range too small to measure, 
and the local disk or dwarf spheroidals, where ancient stars are present but star formation is ongoing at the present epoch. There is also the question of what population we are discussing, when we refer to the bulge. The question of whether the Galaxy even has a bulge was possibly debatable until the $C O B E$ satellite produced a striking image at 2 microns that clearly shows a substantial bulge population (Weiland et al. 1994; Arendt et al. 1994). Does the Galactic Center belong to this population? If it does, then there is evidence that star formation is ongoing today, as has been discussed in this meeting in reviews by Sellgren, Genzel, and Becklin (see also Genzel et al. 1994). And the bulge has RR Lyrae stars (Baade 1951) which are an ancient population. Even if one excludes the Galactic center, there is a concentration of luminous stars within the central $100 \mathrm{pc}$ (Catchpole et al. 1990 ) and there are a large number of Miras with periods in excess of 300 days (Glass et al. 1994).

Of course, the question actually is whether most of the bulge formed at the same time, and if so, when and how? In this review, I am going to discuss progress in 4 approaches toward answering this question. I will start out by stating that even early work (e.g. vanden Bergh, 1971, Rich, 1985) showed that there are few if any young turnoff stars in the bulge. The problem is difficult, and not just because of the extinction, crowding, superposition of stellar populations, and geometrical depth effects that we face in working toward the bulge. It is difficult because the population is older than $5 \mathrm{Gyr}$ and has a narrow age range, features that greatly limit the precision of obvious age indicators like turnoff photometry and population synthesis.

We consider 4 methods that can be used to answer either or both of the questions "when?" and "how?" did the bulge form. The first approach is the frontal assault, age determination from the main sequence turnoff and comparison to globular clusters. We address a related approach (to which we have already alluded), the use of evolved stars such as Miras and RR Lyraes to constrain both the age and age spread in the population. Our third approach focuses more on the question of "how and how long?" than on the "when?". In this approach, we explore the chemical abundance ratios of the bulge stars, which may preserve the record of the type of supernovae (and therefore the timescale) of the enrichment. A fourth approach concerns dynamics, specifically asking what constraints observed dynamics places on formation scenarios. If we demand an age spread, or absolutely no age spread, does this make sense in terms of the observed structure and kinematics of the bulge? 


\section{Turnoff Photometry}

In considering the bulge, one is most interested in the lower latitude fields where the mean metallicity is higher, and classic bulge indicators such as late-type $\mathrm{M}$ giants are found in large numbers (cf. Blanco, 1988). Further, the low-latitude fields are more dominated by bulge population and less likely to suffer contamination by either the disk or the metal poor halo. So while Baade's Window $\left(b=-4^{\circ}\right)$ is the prime location to characterize the bulge, the population is so severely crowded that ground-based work on the main sequence turnoff was confined to higher latitude fields; vanden Bergh (1971), Rich (1985) and Terndrup (1988) found the main sequence population in fields no closer than $-8^{\circ}$. Terndrup (1988) succeeded in fitting isochrones to the turnoff at $-8^{\circ}$ and $-10^{\circ}$ and found an age range of 8-12 Gyr (adjusted to $R_{0}=8 \mathrm{kpc}$. But it could be argued that these higher latitude fields are mostly outside the inner bulge with lower metallicity and perhaps different age. Holtzmann et al. (1993) used the WFPC imager on the Hubble Space Telescope to explore the main sequence turnoff in Baade's Window. As Baum et al. (1993) so eloquently characterized, they found evidence for a "middle-aged" bulge in the Galaxy, perhaps dominated by stars as young as 8 Gyr. Mould (1992) analyzes the WFPC2 luminosity function to pose a credible argument that the bulge might be of order 13 Gyr old and this line of reasoning finds some new support (which is discussed below). While isochrone fitting is debated, Ortolani, Rich, \& Renzini (1994) have spent the last few years obtaining deep images of bulge fieids using the NTT and have succeeded in just reaching the Baade's Window turnoff from the ground. Most importantly, they have verified Holtzmann et al.'s calibration, an issue of some importance given the uncertainties of calibrating HST photometry.

A recent surprise has emerged (at the time of this writing). There is a population of metal rich globular clusters in the bulge; recent high dispersion work by Barbuy et al. (1992) shows that [M/H] for NGC 6553 is -0.2 , a value close to the mean Baade's Window [Fe/H] $(\mathrm{McWilliam} \&$ Rich, 1994). Using HST, Ortolani et al. (1995) obtain turnoff photometry for NGC 6553 and NGC 6528 , an equally metal rich globular cluster. At once, they overcome many of the problems that hamper investigation of the bulge field: geometric depth, abundance spread, and differential reddening. They show that the turnoff to horizontal branch magnitude spread for these clusters is $3.6 \mathrm{mag}$, a value similar to that of other old halo clusters such as 47 Tuc. NTT images of Baade's Window cover a wide enough area to detect clump giants (horizontal branch stars) yet also go deep enough to reach the turnoff. When the luminosity function of the bulge globular clusters is broadened to simulate the depth of the bulge, the two can be aligned 
(without reddening uncertainty) by a force-fit of the clump peaks (due to long-lived horizontal branch stars) in the luminosity function. The result is that the bulge and broadened cluster luminosity functions are identical, and age spreads as small as $10 \%$ at the turnoff appear to be ruled out by the data. At face value, this experiment provides the answer that the bulge was formed all at once (within any reasonable uncertainty) and that it is as old as the halo. However our other approaches (evolved stars, abundances, and kinematics) suggest that the bulge may have had a longer, more complex formation history than Ortolani et al.'s analysis implies. In the end, it is a worthwhile endeavor to seek agreement between all of the various approaches to this problem.

\section{Evolved Stars}

For the reasons cited above, turnoff photometry may fall short as an ideal age indicator in the Galactic bulge. It certainly falls short in M31, M32 and any galaxies more distant. Of great interest is the possibility of using the luminous evolved stars, particularly the AGB, as an age indicator. AGB tip luminosities well in excess of the first giant branch might signal the presence of an intermediate age population (cf. Renzini, 1993). Miras with periods exceeding 300 days have been shown (in the Solar neighborhood) to have disk kinematics, as opposed to the presumably halo population Miras of period 200 days or less. Again, long period Miras at the AGB tip would be the progeny of some kind of massive star population. Merged binaries (blue stragglers) could account for some of these, (Greggio \& Renzini, 1990) although note that most of the Miras in the Sgr I bulge field exceed 300 day period (Glass et al. 1994). If the bulge is old, with a small age range, and approximately Solar metallicity (McWilliam \& Rich, 1994) we have to wonder why its Miras differ so greatly from those of the Solar neighborhood. If surveys of Miras miss the reddest stars, they miss stars of the longest period. In any case, the surveys are presently complete enough that the period distribution is a serious problem. As Renzini (1993) points out, the most luminous giants have potential value in extending the "age ladder" to extragalactic populations. This is provided that such stars do not give misleading results locally. No doubt, the progeny of (massive) blue stragglers must account for some fraction of the population of Miras, but not all of them. So at face value, the presence of both RR Lyrae and Mira variables in the same stellar population would demand an age range, but we still do not know enough about the Miras to settle the question for certain. 


\section{Abundances}

The question "Did the bulge form all at once?" could be taken to mean "Did the bulge form in a violent starburst?" It has long been felt that elliptical and bulge-like systems turn their gas into stars early and rapidly (cf. Matteucci \& Brocato 1990) with consequent enhancement of oxygen and alpha-capture elements. The "smoking gun" for such a process would be to see stars with solar iron abundance and $[0 / \mathrm{Fe}]>0$ and $[\alpha / \mathrm{Fe}]>0]$. Actual high resolution spectroscopy of 11 bulge giants (McWilliam \& Rich, 1994) finds a serious problem: $\mathrm{Mg}$ and $\mathrm{Ti}$, well-known to be alpha-capture elements, are enhanced in the bulge, but $\mathrm{Si}$ and $\mathrm{Ca}$ follow the behavior characteristic of the solar neighborhood. Although difficult to measure, oxygen does not appear to show any dramatic enhancements in these stars (McWilliam, Tomaney, \& Rich, 1995); while their sample is small, other groups working on different bulge giants have also failed to find extreme oxygen enhancements. On the other hand, the $\mathrm{MgH}$ molecule is strong in bulge giants. Rich (1988) used a combination of the $\mathrm{Mg}_{2}$ index and strong Fe lines to estimate abundances of these stars compared to the Solar neighborhood; in retrospect it would appear that the high abundance scale of $[\mathrm{M} / \mathrm{H}]=+0.3$ was due to the apparent enhancement of $\mathrm{Mg}$, which may be present in all bulge stars (Terndrup, Sadler, \& Rich, 1995).

The McWilliam \& Rich study also finds Solar [s/Fe] abundances in the bulge giants. If larger surveys of the bulge continue to find stars with normal s-process abundances, one is inclined to conclude that the bulge formed after an earlier generation of stars. In the extreme halo, the extreme drop of $[\mathrm{Ba} / \mathrm{Eu}]$ at low $[\mathrm{Fe} / \mathrm{H}]$ (Wheeler et al. 1989) is consistent with an early generation of massive star supernovae that occurred before any asmyptotic giant branch star had produced s-process elements in its $\mathrm{He}$ burning shell. Had the starburst event from which the bulge formed been instantaneous $\left(<10^{8} \mathrm{yr}\right)$ one would expect to see only the products of massive star nucleosynthesis in the bulge giants. But this is not the case; the bulge is not identical to the solar neighborhood; the $\mathrm{Mg}$ enhancement does appear to reflect some kind of starburst history. But it seems attractive to consider the event to have been extended in time, also helping to understand the growing evidence for dissipative formation and enrichment.

\section{Structure and Kinematics}

High surface brightness, central concentration, and relative lack of flattening are the distinguishing characteristics of the bulge. Within the effective radius of the bulge, the density is $\approx 1000$ atoms $/ \mathrm{cm}^{3}$ with a dynamical time $\approx 3 \mathrm{Myr}$, and the cooling time is even shorter. The high density and consequent short cooling time have been advanced as arguments that $L_{*}$ 
elliptical galaxies should form quickly (Rees \& Ostriker 1977, Silk 1977). These ideas, along with the $1 / \sqrt{\rho}$ collapse timescale, have caused theorists to favor a rapid, early collapse for spheroids (cf. Renzini 1993). An early collapse and violent starburst is entirely consistent with the bulge metallicity distribution. However, distinguishing characteristic of the bulge is its triaxiality (Blitz \& Spergel, 1990; Dwek et al. 1995) and it is very difficult to come up with an intuitive explanation for how such a scenario makes a triaxial bulge.

The question "Did the bulge form all at once?" might also be construed as asking whether the metal rich and metal poor stars formed at the same time. The work of Rich (1990), Minnitti (1992,1994), and Rich et al. (1995) continues to find the metal rich bulge stars to be more rotation-supported than the metal poor stars. There is also some evidence that the lower metallicity RR Lyrae stars have a higher velocity dispersion than the metal rich bulge stars. The bulge and the extended metal poor halo evidently could not have formed at the same time, "all at once". If we insist that the bulge is as ancient as the oldest stars, we then must allow enough time for the proto-bulge gas to dissipate into the center. Both in its structure and metallicity, the bulge is distinguished from the metal poor halo. Even if these populations formed at the same time, their respective formation processes had to be very different.

\section{Conclusions}

Initial data from a small sample of bulge giants observed at high resolution suggests that the bulge does not show all of the chemical signs of a rapid starburst (cf. Matteucci \& Brocato, 1990). There is continuing evidence as well that the bulge population has correlations between abundances and kinematics. These characteristics suggest that the full formation timescale was no less than $10^{9} \mathrm{yr}$. This is barely compatible with the analysis of Ortolani et al. (1995) which suggests that the largest admissible age spread is $\approx 5 \%$. The population of luminous long period Miras that has been unequivocally established to be present in the bulge is incompatible with a bulge as old as the extreme halo, but has no bearing on the age range of the bulge. The high density of the bulge and correlations between abundances and kinematics are two powerful arguments in favor of a dissipative formation process, one that would have been extended in time. On the other hand, the data are now sufficiently good to rule out the idea that the bulge accumulated slowly from one or more mergers of satellite systems. Another idea for rapid bulge formation is that a massive stellar disk (formed in starburst?) became bar unstable; this structure could have thickened into a triaxial peanut-shaped bulge in < 1 Gyr ( Combes \& Sanders, 1981; Pfenniger \& 
Norman, 1990; Sellwood \& Merritt, 1994). It is not clear whether such a process would preserve correlations between abundances and kinematics. In this last scenario, the bulge structure would have formed suddenly, but the stars comprising that structure could have formed over an extended period. It would appear that the present data support a timescale of order one Gyr for the formation of the bulge, but the chemical signatures of a violent starburst history are only partially present. Yet the most evolved stars stand as a problem, as they have since the subject began: How can RR Lyraes and 300+ day Miras coexist as part of the same population if the age range is narrow? While recent observations have advanced the subject greatly, we can only conclude that the time and timescale of bulge formation remain unsolved problems of the Milky Way.

\section{Acknowledgements}

RMR acknowledges financial support from NASA LTSARP grant NAGW2479 , and helpful discussions about dissipative bulge formation with David Spergel, and discussions with Alvio Renzini and Francesca Matteucci.

\section{References}

Arendt, R.G. et al. 1994, ApJ, 425, 530

Baade, W. 1951, Pub.Obs.Univ.Mich., 10, 7

Barbuy, B., Castro, S., Ortolani, S., \& Bica, E., 1992 AA, 259, 607

Baum, W., Light, R.M., Holtzman, J.A. et al. 1993, in Galactic Bulges, Proc. IAU Symp. 153, Dejonghe H., Habing H.J., eds, Kluwer, Dordrecht, 289

Blanco, V.M., 1988, AJ, 95, 1400

Blitz, L, \& Spergel, D.N., 1991, ApJ, 313, 59

Catchpole, R.M., Whitelock, P.A., \& Glass, I.S. 1990, MNRAS, 252, 210

Combes, F., \& Sanders, R.H. 1981, AA, 96, 154

Dwek, E. et al. 1995 ApJ in press

Glass, I., Whitelock, P.A., Catchpole, R.M., \& Feast, M.W. 1995, MNRAS in press.

Greggio, L., \& Renzini, A., ApJ, 364, 35

Matteucci, F., \& Brocato, E., ApJ, 365, 539

McWilliam, A., \& Rich, R.M. 1994, ApJS, 91, 749

McWilliam, A., Tomaney, A., \& Rich, R.M. 1995, ApJ, submitted

Mould, J.R. 1993 in Science With the Hubble Space Telescope, Baia Chia Workshop

Minnitti, D.M., White, S.D.M., Olszewski, E.W., \& Hill, J.M. 1992, ApJ, 393, L47

Minnitti, D., 1994, Ph.D. thesis, University of Arizona

Ortolani, S., Rich, R.M., \& Renzini, 1994 in preparation

Ortolani, S., Renzini, A., et al. 1995, Nature, Submitted

Pfenniger, D., \& Norman, C., 1990, Ap.J., 313, 391

Rees, M.J., \& Ostriker, J.P. 1977, MNRAS, 179, 541

Renzini, A., 1993, in Galactic Bulges, Proc. IAU Symp. 153, Dejonghe H., Habing H.J., eds, Kluwer, Dordrecht, 391

Rich R.M., 1985, Mem.S.A.It., 56, 23

Rich R.M., 1988, AJ, 95, 828

Rich R.M., 1990, Ap.J., 362, 604.

Rich R.M., 1993, in Galactic Bulges, Proc. IAU Symp. 153, Dejonghe H., Habing H.J., 
eds, Kluwer, Dordrecht, 169

Rich R.M., Terndrup, D.M., \& Sadler, E.M. 1995 in preparation

Sellwood, J.A., \& Merritt, D., 1994, ApJ, 425, 530

Silk, J., 1977, ApJ, 211, 638

Terndrup, D.M., 1988, AJ, 96, 884

Terndrup, D.M., Sadler, E., \& Rich, R.M. 1995, AJ, in press

van den Bergh, S., 1971, AJ, 76, 1082

Weiland, J.L. et al. 1994, ApJ, 425, L81

Wheeler, J.C., Sneden, C., \& Truran, J.W., Jr. 1989, ARAA, 27, 279 